\section{Óbito por dengue como evento sentinela para avaliação da qualidade da assistência: estudo de caso em dois municípios da Região Nordeste, Brasil, 2008}

\author{
Death from dengue fever as a sentinel event for \\ evaluation of quality of healthcare: a case study \\ in two municipalities in Northeast Brazil, 2008
}

1 Instituto de Medicina Integral Prof. Fernando Figueira, Recife, Brasil. 2 Instituto de Higiene

e Medicina Tropical, Universidade Nova de Lisboa, Lisboa, Portugal. 3 Universidade Federal de Pernambuco, Recife, Brasil. 4 Centro de Pesquisas Aggeu Magalhães, Fundação Oswaldo Cruz, Recife, Brasil.

Correspondência A. C. Figueiró

Instituto de Medicina Integral Prof. Fernando Figueira.

Rua José Trajano 170, apto. 301, Recife, PE 51020-320, Brasil.

anaclaudiafigueiro@yahoo. com.br

\begin{abstract}
Despite the existing resources for adequate dengue patient care in the Brazilian healthcare system, the case-fatality rate for the disease is still high in the country. In order to identify factors associated with dengue-related death, this study evaluated quality of care according to the degree of implementation of specific measures, the technical and scientific quality of care, and access to health services in two municipalities (counties) in Northeast Brazil. An evaluative study of the implementation analysis type was performed, with death from dengue as the sentinel event for quality of care. To assess the degree of implementation and quality of care, the study scored the interview criteria and patient chart analysis; access was evaluated by thematic analysis. As for structure and process, the health services were found to be partially adequate (70\%). No geographic or economic barriers were found to explain the occurrence of deaths. Technical and scientific quality failed to achieve adequate levels in the municipalities (46\% and 30\%) or in the specific services, and clinical management of dengue by the health services proved insufficient.
\end{abstract}

Dengue; Health Evaluation; Delivery of Health Care; Sentinel Surveillance
Ana Cláudia Figueiró ${ }^{1}$

Zulmira Maria de Araújo Hartz ${ }^{2}$

Carlos Alexandre Antunes de Brito ${ }^{3}$

Isabella Samico 1

Noêmia Teixeira de Siqueira Filha 1

Gisele Cazarin 1

Cynthia Braga 4

Eduarda Ângela Pessoa Cesse 4

\section{Introdução}

A dengue vem se destacando nas últimas décadas como um problema reemergente de saúde pública, em todo o mundo, tanto pelo número de casos e óbitos, como pelo expressivo contingente de pessoas expostas ao risco de contraí-la. Estima-se que 2,5 bilhões de pessoas, o equivalente a dois quintos da população mundial, estão sob risco de contrair dengue e que ocorram anualmente 50 milhões de casos. Deste total, calculase que 550 mil necessitem de hospitalização e, pelo menos, 20 mil evoluam para óbito 1 .

Segundo dados dos sistemas de informação do Ministério da Saúde, verifica-se tendência crescente dos casos de febre hemorrágica e de óbitos pela doença na última década, com uma taxa média de letalidade de 6,8\% 2 entre 2000 e 2009. A Região Nordeste do Brasil registrou o maior número de casos da enfermidade no país, entre os anos de 1981 a 2007, bem como alta proporção de febre hemorrágica e óbitos por dengue, menor apenas do que o registrado na Região Sudeste, considerando o período de 1990 a 2007 3,4.

Com o objetivo de reduzir a infestação pelo Aedes aegypti, a incidência da dengue e a letalidade por febre hemorrágica causada pela doença, implementou-se no país, em 2002, o Programa Nacional de Controle da Dengue (PNCD) 5. O programa dispõe de dez componentes com atribuições nas três instâncias de gestão do Sistema 
Único de Saúde (SUS), compreendendo ações operacionais de vigilância integrada, entomológica e sobre o meio ambiente; de assistência aos pacientes; de educação em saúde, comunicação e mobilização social. O PNCD compreende, ainda, ações de coordenação, como capacitação dos profissionais; sustentação político-social e monitoramento e avaliação. Entretanto, os dados epidemiológicos têm evidenciado dificuldades no que se refere não só ao desenvolvimento das ações propostas, como também ao alcance dos resultados esperados pelo programa 2 , entre eles, a redução da letalidade por febre hemorrágica causada pela dengue a valores menores ou iguais a $1 \%$, meta prevista para o componente 3 , assistência aos pacientes, do PNCD 5 , objeto do presente estudo.

Embora não se disponha de tratamento específico para a dengue e não se conheçam ainda os mecanismos que predispõem o agravamento dos casos, o choque e o óbito, os quadros graves e mortes pela doença poderiam ser evitados ou reduzidos adotando-se medidas de suporte terapêutico comprovadamente eficazes e disponíveis nos serviços de saúde 6,7 .

$\mathrm{Na}$ avaliação da qualidade da assistência à saúde, tem sido frequente a utilização da abordagem de Donabedian 8,9, compreendendo aspectos de estrutura, processo e resultado. Essa abordagem foi adotada no presente estudo para avaliar o grau de implantação da intervenção quanto à organização das ações e as práticas assistenciais nos serviços. Quanto à adequação no manejo do caso segundo a necessidade dos pacientes, complementou-se com a análise técnico-científica da atenção prestada a estes, observando-se a tecnologia para o nível de atenção, os conhecimentos técnico-científicos atuais, as normas e protocolos, além das práticas e valores culturalmente aceitos 10,11,12. Avaliou-se, ainda, o acesso dos usuários à rede de serviços de saúde, segundo a percepção de sua condição, seus recursos, bem como os obstáculos oferecidos por esses serviços 13 .

Os altos índices de letalidade por dengue e o reconhecimento da responsabilidade direta do setor da saúde na promoção de ações e práticas capazes de evitá-la motivaram a realização desta investigação. O estudo objetivou avaliar a qualidade da assistência aos pacientes que foram a óbito por dengue, em um estado da região Nordeste, no ano de 2007, esperando responder as seguintes perguntas avaliativas: (1) Os serviços de saúde tinham condições de estrutura e processo para assistir os pacientes de dengue, conforme previsto para o seu nível de complexidade? (2) Os pacientes que foram a óbito receberam assistência segundo os padrões de qualidade téc- nico-científica esperado no manejo clínico? (3) A acessibilidade dos pacientes à rede de serviços de saúde influenciou no desfecho da doença?

Assume-se como pressuposto para este estudo que a elevada ocorrência de óbitos por dengue é consequência da baixa qualidade da assistência relacionada às condições organizacionais dos serviços e práticas profissionais quanto ao manejo clínico dos casos. Está relacionada também a outros fatores que não serão tratados no presente artigo, como a gestão municipal do programa e sua influência na organização e articulação com a rede assistencial.

\section{Métodos}

Realizou-se pesquisa avaliativa, tomando-se o óbito por dengue como evento sentinela para avaliar a influência da implementação da intervenção (componente assistência aos pacientes, do PNCD 5) sobre os efeitos observados 14. A estratégia de pesquisa utilizada foi o estudo de caso com níveis de análise imbricados 15.

Constitui evento sentinela uma ocorrência inesperada envolvendo óbito ou sérios danos físicos ou psicológicos, ou o risco de vir a acontecer, sendo sua ocorrência um marcador da qualidade ou da efetividade do sistema de serviços de saúde ${ }^{16}$. Nesse sentido, um único caso de óbito por dengue, tratando-se de doença evitável com os recursos disponíveis, pode ser tomado como evento sentinela. A denominação de "sentinela" sinaliza a necessidade de investigação e resposta imediata. Tais eventos têm sido utilizados, tal como proposto Rustein et al. 17, em vários países para o monitoramento da qualidade da atenção ao paciente 18,19,20.

A trajetória assistencial 21 do paciente pela rede de saúde durante a ocorrência de um evento sentinela permite identificar os serviços utilizados e, a partir da investigação do evento, apreciar a adequação das condições de funcionamento e qualidade da assistência prestada no sistema de saúde. O registro e a análise dos óbitos por dengue como eventos sentinelas constituem, assim, uma importante oportunidade para indicar os problemas que levaram à sua ocorrência e as possibilidades de mudanças em processos organizacionais, visando a aumentar a segurança dos pacientes e prevenir episódios similares no futuro.

A seleção do local do estudo considerou tratar-se de área prioritária para implantação do PNCD. No estado da Região Nordeste eleito para o estudo, foram selecionados o Município A, a capital, e o Município B, o de maior ocorrência de óbito por dengue, excetuando-se a capital. Os 
serviços de saúde visitados pelo paciente durante o episódio da doença foram identificados seguindo a sua trajetória assistencial desde o aparecimento dos primeiros sintomas até o desfecho do evento $21,22,23$.

Segundo dados do Ministério da Saúde, ocorreram 14 óbitos por dengue em 2007, ano-base do estudo, nos dois municípios selecionados, sendo todos hospitalares. Desse total de óbitos notificados, dez ocorreram em quatro hospitais, sendo dois localizados no Município A e dois, no B. Dois dos quatro hospitais eram definidos como referência da rede estadual de saúde para tratamento de casos graves de dengue, o que implicaria capacitação dos profissionais nos procedimentos normatizados para assistência aos pacientes. Os outros dois são serviços de referência municipal e estadual para saúde da criança. Os prontuários médicos desses hospitais constituíram o ponto de partida para identificação da trajetória do paciente na rede de serviços de saúde. Dos dez óbitos, cinco compuseram a amostra final do estudo; os demais foram excluídos, pois não foi possível localizar a família pelo endereço no prontuário (três casos) e o município de residência da família era muito distante da capital do estado (Município A) e do Município B, não utilizando a rede de serviços dos municípios selecionados (dois casos).

O estudo empregou métodos qualitativos e quantitativos na coleta de dados. A trajetória assistencial dos óbitos foi identificada por meio de entrevistas semiestruturadas com os familiares dos pacientes, contemplando o desenvolvimento da doença desde os primeiros sintomas até o desfecho do caso, os serviços visitados e a percepção da qualidade da assistência prestada. Para avaliar o grau de implantação das ações e práticas de atenção à saúde nos serviços, foram empregados questionários estruturados complementados por questões abertas. Na dimensão da qualidade técnico-científica da assistência, utilizou-se roteiro estruturado para análise documental dos prontuários médicos dos óbitos por dengue. Essas duas análises foram utilizadas para responder as primeira e segunda questões do estudo, respectivamente; a terceira foi respondida com as informações dos familiares quanto aos eventos avaliados.

Os critérios e indicadores de avaliação, assim como os parâmetros de julgamento e de classificação dos serviços quanto à qualidade da assistência, foram definidos com base nas publicações técnicas e normativas do Ministério da Saúde 5,12. A opção pelas publicações e normativas oficiais deveu-se ao fato de que elas têm sido continuamente utilizadas nas atividades de capacitação dos profissionais de saúde respon- sáveis pela assistência aos pacientes de dengue, sendo presumidamente de amplo conhecimento entre esses profissionais.

Após a construção de uma primeira matriz de análise e julgamento, procedeu-se à consulta de opinião de especialistas no tratamento de casos de dengue (dois) e profissionais dos serviços de saúde (dois), visando ao consenso no que diz respeito aos aspectos descritos para as condições esperadas na assistência adequada aos pacientes. A matriz foi, então, finalizada, após revisão das observações e sugestões dos consultados. Para a análise dos prontuários médicos, partiuse do instrumento elaborado pelo Ministério da Saúde para investigação de casos graves e óbitos por dengue, o qual foi testado e adaptado a fim de se realizar o presente estudo; na sua validação, foram utilizados casos graves de dengue internados em hospital de referência do estado, no mesmo período estudado. Os aspectos contemplados nos instrumentos para avaliação do grau de implantação estão descritos na Tabela 1.

Foram entrevistados profissionais de diferentes categorias e serviços de saúde, segundo a complexidade e o setor, selecionados de acordo com o dia da semana e o turno de trabalho; buscou-se contemplar dias úteis e finais de semana, além de horário diurno e noturno. Os questionários estruturados para médicos, enfermeiros, chefes de serviço e de setor e profissionais dos núcleos de epidemiologia foram respondidos pelos próprios entrevistados após explicitação dos objetivos do estudo. Os questionários para os profissionais de apoio laboratorial e as entrevistas semiestruturadas com familiares foram realizadas por profissionais de nível superior com experiência em pesquisa. A avaliação dos prontuários foi realizada por médico clínico com reconhecida expertise em dengue. Dados epidemiológicos referentes à letalidade por dengue para cada hospital e município foram utilizados para avaliar a influência da intervenção nos efeitos observados.

As entrevistas estruturadas e a análise dos prontuários tiveram seus dados inseridos em planilhas Excel (Microsoft Corp., Estados Unidos), posteriormente categorizados para pontuação e classificação dos serviços. A análise dos dados se fez pela soma dos pontos obtidos para cada critério, sendo a classificação final calculada pela somatória das pontuações atingidas em cada dimensão (grau de implantação $=\Sigma$ pontos alcançados / pontos esperados X 100).

As entrevistas semiestruturadas feitas com os familiares foram gravadas e transcritas para análise. Recorreu-se à técnica de análise temática 24 , tomando-se como unidade de contexto o conjunto das entrevistas, a fim de se identificarem e interpretarem os temas de interesse. Para 
Tabela 1

Definição dos critérios para avaliação da qualidade da assistência aos casos de dengue. Municípios A e B. Região Nordeste, Brasil, 2008.

Organização dos serviços e práticas de saúde

Critérios

Assistência hospitalar aos pacientes

Estrutura

Processo

Diagnóstico e tratamento da dengue e da febre hemorrágica causada pela doença, conforme previsto nas normas técnicas do Ministério da Saúde

Núcleo Hospitalar de Epidemiologia

Estrutura

Processo
Adequação de profissionais para desenvolvimento das práticas

Suficiência de leitos em período epidêmico e

interepidêmico

Adequação dos recursos materiais (físico, equipamentos,

medicamentos, impressos e exames laboratoriais)

Presença nos consultórios e adoção pelo serviço de normas

técnicas/protocolos para assistência aos casos de dengue

Aplicação dos recursos financeiros

Profissionais capacitados

Influência da capacitação na prática de saúde

Conhecimento e utilização, pelos profissionais, das normas

técnicas para diagnóstico e tratamento

Conhecimento, pelos profissionais, das rotinas e fluxos do

NEPI

Conhecimento da rotina do apoio laboratorial e opinião

sobre ela por parte dos profissionais

Disponibilidade e uso de materiais educativos

Recebimento e consulta do material do Ministério da Saúde

Critérios para diagnóstico da dengue

Critérios para diagnóstico da febre hemorrágica

Solicitação de exames para confirmação de casos de

dengue

Realização da prova do laço e situações a que se aplica

Hidratação prescrita, supervisionada e reavaliada, conforme

recomendado pelo Ministério da Saúde

Utilização e correta descrição do estadiamento clínico da

dengue preconizado pelo Ministério da Saúde

Dificuldades para diagnóstico clínico da dengue

Opinião sobre o material educativo

Percepção dos profissionais quanto às condições de

trabalho no setor e ocorrência de óbitos por dengue

Existência do NEPI no hospital com normas de

funcionamento e fluxo definido

Adequação dos profissionais em atuação (quantidade e

qualidade)

Adequação dos recursos materiais (físico, equipamento,

material, insumos)

Recursos financeiros aplicados como previsto

Busca ativa e passiva para detecção de casos de dengue Notificação e investigação de casos e óbitos de dengue

Discussão dos casos e dos óbitos de dengue com os setores

Produção de informação regular

Atuação integrada ao laboratório

Decisões tomadas no serviço, influenciadas pela ação do NEPI

Percepção dos profissionais quanto às condições de trabalho no setor 


\begin{tabular}{|c|c|}
\hline \multicolumn{2}{|c|}{ Organização dos serviços e práticas de saúde } \\
\hline Critérios & \\
\hline \multicolumn{2}{|l|}{ Apoio laboratorial } \\
\hline \multirow[t]{3}{*}{ Estrutura } & $\begin{array}{l}\text { Adequação dos profissionais em atuação (quantidade e } \\
\text { qualidade) }\end{array}$ \\
\hline & $\begin{array}{l}\text { Adequação dos recursos materiais (físico, equipamento, } \\
\text { material, insumos) }\end{array}$ \\
\hline & $\begin{array}{l}\text { Existência e uso de normas de funcionamento e fluxo } \\
\text { definido }\end{array}$ \\
\hline \multirow[t]{6}{*}{ Processo } & Adequação da quantidade e disponibilidade dos exames \\
\hline & para atendimento aos casos de dengue \\
\hline & Adequação do tempo médio entre a solicitação e entrega \\
\hline & dos resultados dos exames \\
\hline & Percepção dos profissionais quanto às condições de \\
\hline & trabalho no setor \\
\hline \multicolumn{2}{|l|}{ Qualidade técnico-científica } \\
\hline \multirow[t]{9}{*}{ Adequação clínica no manejo dos casos } & Sinais de alarme e choque no prontuário \\
\hline & Estadiamento clínico da dengue preconizada pelo \\
\hline & Ministério da Saúde no prontuário \\
\hline & Hidratação prescrita, supervisionada e reavaliada conforme \\
\hline & recomendado pelo Ministério da Saúde no prontuário \\
\hline & Solicitação de exames adequada e oportuna para \\
\hline & reavaliação do paciente no prontuário \\
\hline & Decisões tomadas com base nas informações dos exames \\
\hline & laboratoriais \\
\hline \multicolumn{2}{|l|}{ Acessibilidade e percepção dos usuários } \\
\hline \multirow{4}{*}{$\begin{array}{l}\text { Acessibilidade ao serviço/Integração da rede de serviços/Barreiras organizacionais, } \\
\text { sociais e geográficas }\end{array}$} & Condições de acesso ao serviço de saúde \\
\hline & Tempo de espera \\
\hline & Assistência realizada por médico \\
\hline & Referência e contrarreferência \\
\hline \multirow[t]{4}{*}{ Percepção dos usuários quanto à qualidade da assistência prestada } & Procedimentos realizados \\
\hline & Respeito ao paciente \\
\hline & Acompanhamento ao paciente \\
\hline & Opinião sobre atendimento \\
\hline
\end{tabular}

NEPI: Núcleo de Epidemiologia do Serviço de Saúde.

apresentação do conteúdo das entrevistas, estas foram identificadas como: Ent.1, Ent.2, ... Ent.N. Os efeitos da intervenção, expressos nas taxas de letalidade por dengue, foram calculados por hospital de ocorrência do óbito e município.

O presente estudo foi aprovado pelo Comitê de Ética em Pesquisas em Seres Humanos do Instituto de Medicina Integral Prof. Fernando Figueira (protocolo de pesquisa no. 646/07).

\section{Resultados}

\section{Grau de implantação da organização dos \\ serviços e práticas profissionais e da qualidade técnico-científica}

O resultado da análise do grau de implantação das condições de organização e práticas profissionais dos serviços avaliados, assim como da qualidade técnico-científica destes, encontra-se na Tabela 2. Ao final da tabela, descreve-se a taxa de letalidade por dengue nos serviços de saúde e municípios, a qual será tomada como efeito da intervenção. Os resultados derivados das questões abertas estão apresentados na Tabela 3, como principais aspectos positivos e negativos referentes à assistência hospitalar aos pacientes, vigilância epidemiológica e apoio laboratorial. 
Tabela 2

Grau de implantação dos serviços de saúde dos Municípios A e B. Região Nordeste, Brasil, 2008.

\begin{tabular}{|c|c|c|c|c|c|c|c|c|c|c|}
\hline \multicolumn{11}{|c|}{ Dimensão: organização dos serviços e práticas de saúde } \\
\hline \multirow[t]{2}{*}{ Componentes } & \multirow[t]{2}{*}{ Critérios/Indicadores } & \multicolumn{5}{|c|}{ Município A } & \multicolumn{4}{|c|}{ Município B } \\
\hline & & HG, A & $H R, A$ & $\mathrm{PC}, \mathrm{A}$ & UBS, A & $\mathrm{Gl}$ * & HR, B & HM, B & $\mathrm{PC} 2, \mathrm{~B}$ & $\mathrm{Gl}$ * \\
\hline \multirow[t]{3}{*}{$\begin{array}{l}\text { Assistência aos } \\
\text { pacientes }\end{array}$} & $\begin{array}{l}\text { Estrutura } \\
\text { RH, instalações, equipamentos e } \\
\text { materiais; Normas, protocolos e } \\
\text { rotinas; Plano de contingência }\end{array}$ & $\begin{array}{c}48 / 70= \\
68,6 \%\end{array}$ & $\begin{array}{c}53 / 70= \\
75,7 \%\end{array}$ & $\begin{array}{l}55 / 65= \\
84,6 \%\end{array}$ & $\begin{array}{c}29 / 65= \\
44,6 \%\end{array}$ & $68,4 \%$ & $\begin{array}{c}50 / 70= \\
71,4 \%\end{array}$ & $\begin{array}{c}31 / 70= \\
44,3 \%\end{array}$ & $\begin{array}{c}24 / 25= \\
96,0 \%\end{array}$ & $70,6 \%$ \\
\hline & $\begin{array}{l}\text { Processo } \\
\text { Conhecimento e uso das } \\
\text { normas; Capacitação em } \\
\text { dengue; NEPI e apoio } \\
\text { laboratorial; Material educativo; } \\
\text { Integração dos serviços }\end{array}$ & $\begin{array}{c}28 / 50= \\
56,0 \%\end{array}$ & $\begin{array}{c}37 / 50= \\
74,0 \%\end{array}$ & $\begin{array}{c}33 / 35= \\
94,3 \%\end{array}$ & $\begin{array}{c}32 / 35= \\
91,4 \%\end{array}$ & $78,9 \%$ & $\begin{array}{c}29 / 50= \\
58,0 \%\end{array}$ & $\begin{array}{c}33 / 50= \\
66,0 \%\end{array}$ & $\begin{array}{l}25 / 25= \\
100,0 \%\end{array}$ & $74,7 \%$ \\
\hline & $\begin{array}{l}\text { Diagnóstico e tratamento da } \\
\text { dengue e da febre hemorrágica } \\
\text { causada pela doença } \\
\text { Critérios diagnósticos, } \\
\text { casos suspeitos, solicitação } \\
\text { de exames, prova do laço, } \\
\text { hidratação, estadiamento }\end{array}$ & $\begin{array}{c}19 / 25= \\
76,0 \%\end{array}$ & $\begin{array}{c}17 / 25= \\
68,0 \%\end{array}$ & $\begin{array}{c}17 / 25= \\
68,0 \%\end{array}$ & $\begin{array}{c}13,5 / 25= \\
54,0 \%\end{array}$ & $66,5 \%$ & $\begin{array}{c}16,5 / 25= \\
66,0 \%\end{array}$ & $\begin{array}{c}11 / 25= \\
44,0 \%\end{array}$ & $\begin{array}{c}15,5 / 25= \\
62,0 \%\end{array}$ & $58,7 \%$ \\
\hline $\begin{array}{l}\text { Vigilância } \\
\text { epidemiológica }\end{array}$ & $\begin{array}{l}\text { Existência do NEPI ou vigilância } \\
\text { epidemiológica; Adequação: } \\
\text { RH, materiais e financeiros; } \\
\text { Notificação e investigação de } \\
\text { casos; Produção de informação }\end{array}$ & $\begin{array}{c}49 / 55= \\
89,1 \%\end{array}$ & $\begin{array}{c}38 / 55= \\
69,1 \%\end{array}$ & $\begin{array}{c}11 / 15= \\
73,3 \%\end{array}$ & $\begin{array}{l}7 / 15= \\
46,7 \%\end{array}$ & $69,5 \%$ & $\begin{array}{c}30 / 55= \\
54,5 \%\end{array}$ & $\begin{array}{c}38 / 55= \\
69,1 \%\end{array}$ & $\begin{array}{c}5 / 5= \\
100,0 \%\end{array}$ & $74,5 \%$ \\
\hline Apoio laboratorial & $\begin{array}{l}\text { Norma de funcionamento e } \\
\text { fluxo; Adequação: RH, materiais } \\
\text { e financeiros; Quantidade e } \\
\text { disponibilidade de exames; } \\
\text { Tempo médio entre a solicitação } \\
\text { e entrega dos resultados dos } \\
\text { exames }\end{array}$ & $\begin{array}{c}58 / 80= \\
72,5 \%\end{array}$ & $\begin{array}{c}43 / 80= \\
53,7 \%\end{array}$ & NSA & NSA & $63,1 \%$ & $\begin{array}{c}57 / 80= \\
71,3 \%\end{array}$ & $\begin{array}{c}63 / 80= \\
78,8 \%\end{array}$ & NSA & $75,0 \%$ \\
\hline $\begin{array}{l}\text { Resultado da } \\
\text { dimensão }\end{array}$ & & $\begin{array}{l}202 / 280 \\
=72,1 \%\end{array}$ & $\begin{array}{l}188 / 280 \\
=67,1 \%\end{array}$ & $\begin{array}{c}116 / 140= \\
82.9 \%\end{array}$ & $\begin{array}{c}81,5 / 140 \\
=58,2 \%\end{array}$ & $70,1 \%$ & $\begin{array}{c}182,5 / 280 \\
=65,2 \%\end{array}$ & $\begin{array}{c}177 / 280= \\
63,2 \%\end{array}$ & $\begin{array}{c}69,5 / 80= \\
86,9 \%\end{array}$ & $71,8 \%$ \\
\hline \multicolumn{11}{|c|}{ Dimensão: qualidade técnico-científica } \\
\hline \multirow[t]{2}{*}{ Componentes } & \multirow[t]{2}{*}{ Critérios/Indicadores } & \multicolumn{5}{|c|}{ Município A } & \multicolumn{4}{|c|}{ Município B } \\
\hline & & HG, A & $\mathrm{HR}, \mathrm{A}$ & $\mathrm{PC}, \mathrm{A}$ & UBS, A & Gl * & HR, B & HM, B & PC 2, B & Gl * \\
\hline Manejo de casos & $\begin{array}{l}\text { Registro de sinais de alarme e } \\
\text { choque; Estadiamento clínico } \\
\text { preconizado; Hidratação: } \\
\text { prescrita, supervisionada } \\
\text { e reavaliada conforme o } \\
\text { Ministério da Saúde; } \\
\text { Solicitação de exames } \\
\text { adequada e oportuna para } \\
\text { reavaliação do paciente; } \\
\text { Decisões tomadas pelos } \\
\text { profissionais usando os exames } \\
\text { laboratoriais }\end{array}$ & $\begin{array}{l}7 / 25= \\
28,0 \%\end{array}$ & $\begin{array}{c}16 / 25= \\
64,0 \%\end{array}$ & $\begin{array}{l}\text { Prontuário } \\
\text { não } \\
\text { avaliado }\end{array}$ & $\begin{array}{c}\text { Prontuário } \\
\text { não } \\
\text { avaliado }\end{array}$ & $46,0 \%$ & $\begin{array}{c}11 / 25= \\
44,0 \%\end{array}$ & $\begin{array}{l}\text { Prontuário } \\
\text { não } \\
\text { avaliado }\end{array}$ & $\begin{array}{l}4 / 25= \\
16,0 \%\end{array}$ & $30,0 \%$ \\
\hline $\begin{array}{l}\text { Taxa de letalidade } \\
\text { por dengue }\end{array}$ & & $3,1 \%$ & $1,5 \%$ & - & - & $3,6 \%$ & $12,0 \%$ & $50,0 \%$ & NSA & $14,9 \%$ \\
\hline
\end{tabular}

* Grau de implantação: 80,0\% ou mais = Adequado; 79,9\% a 60,0\% = Parcialmente adequado; 59,9\% a 40,0\% = Pouco adequado; $\leq 39,0 \%$ = Não adequado. HG, A: Hospital Geral, Município A; HM, B: Hospital Municipal, Município B; HR, A: Hospital de Referência, Município A; HR, B: Hospital de Referência, Município B; NEPI: Núcleo de Epidemiologia do Serviço de Saúde; NSA: não se aplica; PC, A: Policlínica, Município A; PC 2, B: Policlínica, Município B; UBS, A = Unidade Básica de Saúde, Município A. 
Aspectos positivos e negativos do grau de implantação das ações para assistência aos pacientes de dengue, nos serviços de saúde dos municípios A e B. Região Nordeste, Brasil, 2008.

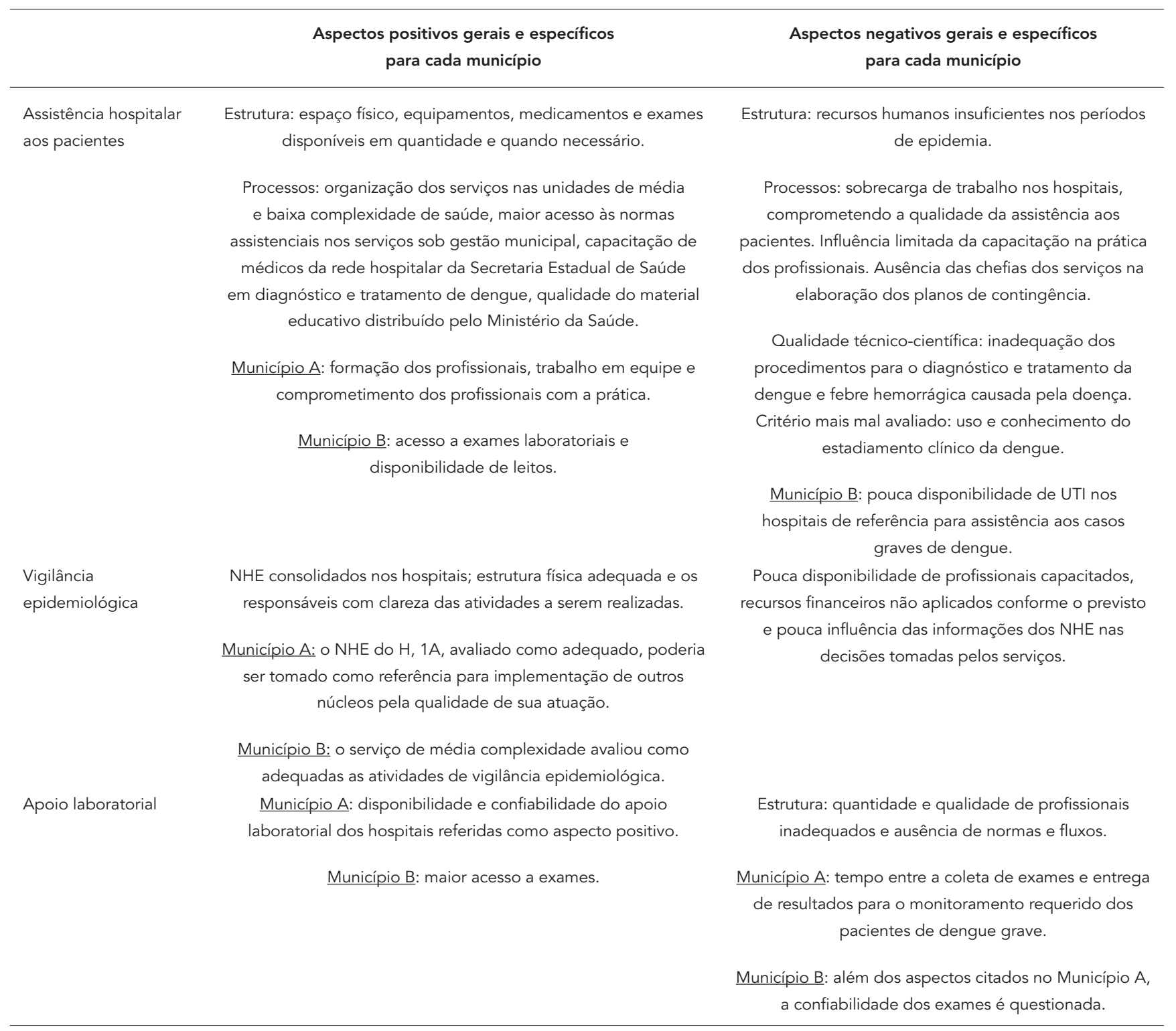

NHE: Núcleo Hospitalar de Epidemiologia.

Os níveis de adequação quanto à estrutura e processo da assistência aos pacientes, a organização da vigilância epidemiológica e do apoio laboratorial não variaram entre os dois municípios. Ambos foram classificados como parcialmente adequados, com valores de $70,1 \%$ e $71,8 \%$ para os municípios A e B, respectivamente; houve também pouca variação ao se comparar cada serviço de saúde. Porém, ao discriminar cada componente, os valores mais baixos foram encontrados para os processos referidos ao diagnóstico e tra- tamento da dengue, bem como febre hemorrágica causada pela doença, $66,5 \%$ correspondendo a parcialmente adequado no Município A e 58,7\%, pouco adequado, no Município B.

Os aspectos favoráveis relacionados a essa dimensão, conforme descrito na Tabela 3, referiram-se, principalmente, à boa disponibilidade dos recursos e à capacitação realizada para os profissionais de saúde sobre manejo clínico da dengue, no ano seguinte aos óbitos avaliados. No entanto, a quantidade insuficiente de profis- 
sionais, principalmente médicos, nos períodos epidêmicos, compromete a assistência aos pacientes, na opinião destes.

Entre as condições observadas como insatisfatórias, as quais, potencialmente, podem contribuir para uma baixa qualidade da assistência, estão: (1) conhecimento e uso das normas e protocolos de orientação do manejo clínico da dengue pelos profissionais; (2) apoio laboratorial, quanto ao tempo de espera para recebimento dos resultados dos exames e confiança dos profissionais nos seus resultados.

Assistência segundo os padrões de qualidade técnico-científica esperados no manejo dos casos

Verificou-se que a assistência aos pacientes não alcançou o nível de adequação esperado em qualquer dos serviços avaliados. $\mathrm{O}$ serviço mais bem avaliado foi classificado como parcialmente adequado (HR, A) e dois serviços foram avaliados como não adequados. Entre os critérios analisados, aquele com mais baixa pontuação foi o estadiamento da doença, procedimento fundamental no diagnóstico e reconhecimento da gravidade do caso. Constatou-se, ainda, a pouca utilização dos exames clínicos para a reavaliação adequada do paciente e, consequentemente, pa- ra as decisões dos profissionais na condução do tratamento.

Nos prontuários, observou-se a insuficiência das informações registradas e algumas situações recorrentes. Entre elas, destacam-se: falha no registro/pesquisa de sinais vitais básicos; sinais de alarme não pesquisados/registrados; ausência de referência ao estadiamento clínico; exames complementares não solicitados ou solicitação não realizada oportunamente e nos intervalos recomendados; volumes de hidratação frequentemente inferiores ao preconizado; reavaliações clínicas realizadas com intervalos bem superiores aos recomendados para pacientes que necessitam de hidratações volumosas e com risco de evoluir para o choque; pacientes transferidos sem prontuário de encaminhamento.

Pela trajetória dos casos, apresentada na Figura 1, verifica-se que quatro dos cinco óbitos eram residentes do Município B, onde iniciaram a busca pelos serviços de saúde. A baixa qualidade técnico-científica desde o primeiro serviço procurado se mantém na continuidade da assistência na rede municipal. Uma vez que o município conta com um hospital estadual de referência para casos graves de dengue, esperava-se que os estes casos fossem assistidos nesse serviço; no entanto, pacientes foram incorretamente encaminhados para hospitais na capital do estado.

Figura 1

Trajetória assistencial dos óbitos nos municípios A e B. Região Nordeste, Brasil, 2008.
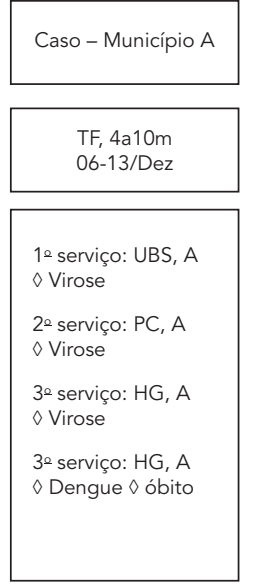
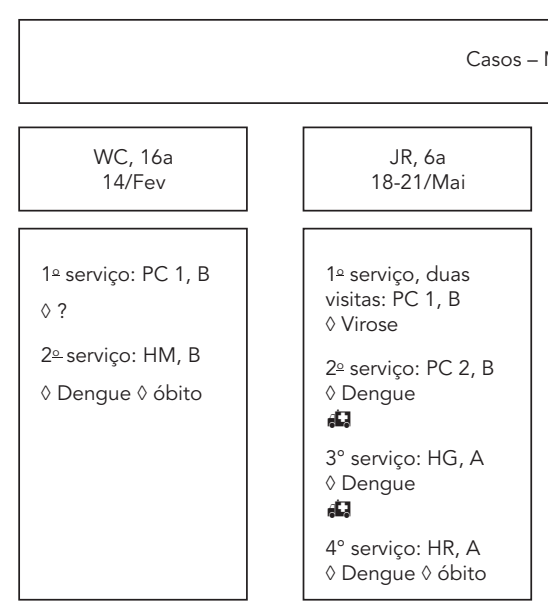

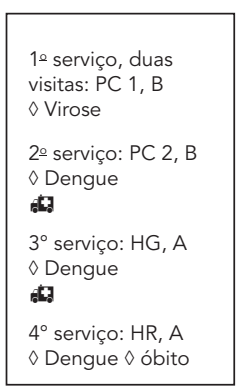

Casos - Município B

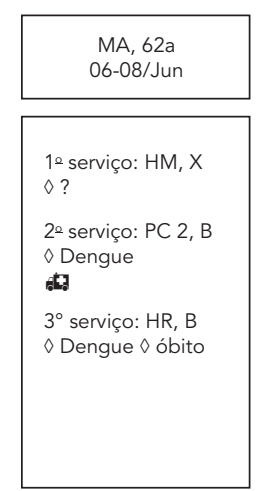

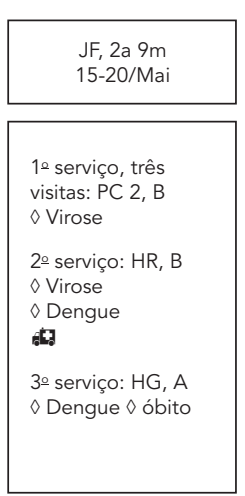

HG, A: Hospital geral, Município A; HM, B: Hospital Municipal, Município B; HM, X: Hospital Municipal, outro município; HR, A: Hospital de Referência, Município A; HR, B: Hospital de Referência, Município B; PC, A: Policlínica, Município A; PC 1, B: Policlínica 1, Município B; PC 2, B: Policlínica 2, Município B; UBS, A: Unidade Básica de Saúde, Município A;

?: Ausência de informação; Transferência do paciente por ambulância. 
Acessibilidade dos pacientes à rede de serviços de saúde

O acesso geográfico à rede de serviços de saúde, na percepção dos familiares de pacientes que foram a óbito, não se constituiu um problema na maioria dos casos avaliados. Os pacientes, em sua maior parte, procuraram os serviços de saúde nos primeiros dias de sintoma da doença, sendo atendidos no mesmo dia e sempre por profissional médico. Barreiras econômicas, como dificuldade financeira para o transporte do paciente ou de acompanhante entre os serviços de saúde, foram pouco referidas pelos usuários.

O aspecto organizacional foi considerado insatisfatório pelos familiares quanto ao tempo de espera para a consulta, ao não funcionamento do serviço em fim de semana e à inadequação do acolhimento, principalmente pelo não reconhecimento da gravidade do caso. Em nenhum dos casos a dengue foi diagnosticada na primeira visita ao serviço de saúde, como pode ser observado na Figura 1.

Os problemas mais relevantes para os familiares referiram-se à resolubilidade do serviço, como revelam as falas que seguem:

"Em uma semana ele já tinha ido duas vezes pra Policlínica $1 B$ [PC 1, B] e os médicos só diziam que era virose. Com oito dias, ele foi piorando $e$ nós levamos na Policlínica 2B. Só diziam que era virose. Ele só foi piorando..." (Ent.2)

"Dois dias depois que ele começou doente [e] eu levei ele pra Policlínica 2B [PC 2, B]. Eu fui e voltei três vezes $e$ [o médico] só dizia que era uma virose. A única coisa que passou era paracetamol e mandou ficar sem roupa. Toda vez não foi feito nenhum exame" (Ent.4).

Os familiares apontaram, também, inadequação do encaminhamento dos pacientes para serviços de maior complexidade. A decisão de buscar esses serviços foi, na maioria das vezes, iniciativa do acompanhante do paciente e não uma orientação do profissional, de acordo com o diagnóstico e reconhecimento do agravamento da doença. Esse resultado se expressa na citação a seguir:

"No meio da noite, eu chamei o médico. Ele disse: 'Vai pra casa, vou liberar'. Outra mulher que estava lá disse assim: 'Não leve esse menino pra casa não, que o meu filho estava assim e foi dengue...' À 1h da manhã [o] exame chegou. Aí o médico disse: 'Esse menino vai pro Hospital $1 A[\mathrm{H}$ 1, A] agora, porque esse menino está com dengue hemorrágico." (Ent.2)

A mesma inadequação da assistência, quanto ao diagnóstico, manejo clínico e encaminhamento, foi percebida pelos familiares no hospital de referência para casos graves da doença, do Município B. Esse problema pode ser constatado no relato a seguir:

"Fui pro [hospital] regional por minha conta porque o doutor dizia assim: 'Lá é só pra caso grave. Quem me atendeu foi a enfermeira (...) meu filho ficou no soro o dia todo; eu perguntei o que estava dando pra ele e ela dizia que era [paraceta$\mathrm{mol}$. Quando ele reclamava de dor de barriga, ela dizia assim: 'Leva ele no banheiro'. Aí, quando ela colocou o supositório, é que viu o sangue. Pediu o exame da barriga, mas ninguém viu o resultado, só viu quando teve sangue (...). Aí ela chamou a médica que disse pra mandar direto pro [HR2], que era dengue hemorrágico." (Ent.4)

O encaminhamento realizado pelo serviço, em ambulância, foi tardio nos dois casos evidenciados acima. Este é um dos problemas citados pelos profissionais dos hospitais da capital do estado e diz respeito às situações que podem ter contribuído para os óbitos por dengue no serviço.

\section{Discussão}

No que se refere ao componente 3 (assistência aos pacientes) do PNCD, a análise imbricada (diferentes níveis de atenção, setores assistenciais, categorias profissionais e usuário) dos cinco casos de óbito por dengue nos municípios estudados, tomados como evento sentinela para avaliação da qualidade da atenção prestada aos pacientes, encontrou que esse componente não alcançou os resultados esperados pelo programa. O efeito maior esperado, a taxa de letalidade por dengue menor que $1 \%$, segundo as normais atuais 25 , não foi alcançado em nenhum dos hospitais avaliados.

Este resultado nos municípios e respectivos serviços de saúde avaliados não pode ser creditado diretamente a problemas referentes à estrutura e ao processo de organização das ações na rede de saúde, visto que os serviços apresentavam condições, no mínimo, parcialmente adequadas para assistência aos pacientes em seu nível de complexidade. Outras avaliações do grau de implantação de ações programáticas em unidades básicas de saúde e hospitais têm identificado situação semelhante 26,27,28. Há indícios de que o grau de implantação dos programas de saúde tem sido, ao menos em parte, apropriado quanto à estrutura e aos processos, não se constituindo no principal problema para alcance dos resultados.

Essa situação relativamente favorável verificada no presente estudo poderia ser percebida distintamente se a coleta de dados ocorresse em 
período de epidemia. Nessas ocasiões, na opinião da maioria dos profissionais entrevistados, o quantitativo de médicos é apontado como insuficiente para a demanda, com repercussão importante na qualidade da atenção aos pacientes. Contudo, os demais aspectos organizativos não foram apontados como problema para assistência aos pacientes, inclusive em período epidêmico.

Quando se observa a relação entre o grau de implantação do componente assistência aos pacientes, do PNCD, no que diz respeito às dimensões organização dos serviços, qualidade técnico-científica no manejo clínico dos óbitos e resultados esperados dessa intervenção, verificase coerência entre a classificação alcançada nas dimensões e o resultado nas taxas de letalidade pela doença nos serviços e municípios. O hospital de referência do Município A, mais bem avaliado nos parâmetros referidos, foi o serviço com menor taxa de letalidade. Por sua vez, a taxa de letalidade do Município A, com melhores resultados nas dimensões avaliadas, foi menor do que a do Município B.

O resultado encontrado no hospital de referência do Município A seria o esperado para os demais serviços de saúde, uma vez que estavam disponíveis, em todos os serviços avaliados, as condições necessárias para assistir adequadamente os casos de dengue e de febre hemorrágica causada pela doença. Portanto, o que se observa nos achados são problemas relacionados, principalmente, ao manejo clínico dos casos. Outros países que viveram epidemias de dengue com altas taxas de letalidade chegaram à mesma conclusão: a aplicação dos conhecimentos e utilização dos recursos disponíveis no país permitiria classificar e reconhecer precocemente os sinais de gravidade do quadro clínico, evitando o desfecho indesejado 6,7,29.

Quanto à acessibilidade, relativa aos aspectos geográficos e econômicos, o acompanhamento das trajetórias assistenciais dos pacientes pelos serviços de saúde permitiu identificar os pontos críticos da rede, que, em seu conjunto, são responsáveis pela ocorrência do óbito ${ }^{30}$. Essas trajetórias indicaram que, apesar de dificuldades verificadas, o acesso à rede não se constituiu em um problema que justificasse as mortes. Os problemas se mostraram, sobretudo, quanto ao diagnóstico e tratamento adequado da doença. Situação semelhante foi observada em estudos sobre o acesso à assistência e a qualidade desta nos casos de óbito infantil em municípios da Região Nordeste do Brasil, onde, apesar das dificuldades relativas à acessibilidade, os problemas se concentraram principalmente na baixa resolubilidade da assistência 21,31.
O manejo clínico dos casos nos hospitais de ocorrência dos óbitos avaliados, em especial, os serviços do Hospital Geral do Município A (HG, A) e do Hospital de Referência do Município B (HR, B), apresentou-se como influência direta sobre essas mortes. Pode-se observar padrão semelhante na condução dos casos analisados: as recomendações do Ministério da Saúde 12 para o manejo dos casos de dengue, as quais permitiriam detectar precocemente os casos graves e realizar o manejo clínico adequado a fim de evitar um desfecho desfavorável, não foram seguidas.

De acordo com os registros nos prontuários avaliados, os pacientes com dengue foram, aparentemente, tratados segundo a rotina para pacientes com outras doenças febris agudas (virose, pneumonia); uma observação constante foi o procedimento quanto ao volume de hidratação, em geral inferior ao recomendado para os casos avaliados. Conforme se verificou nesta investigação, essa conduta pode estar relacionada ao pouco uso da dosagem do hematócrito, elemento importante na orientação da hidratação.

A organização de uma rede de serviços de saúde, desde a atenção básica, com fluxo definido para os diferentes níveis de complexidade, apoio laboratorial e presença de médicos, enfermeiros e demais profissionais capacitados, é medida reconhecida como necessária para evitar a elevada ocorrência de óbitos por dengue, seja na América Latina, seja no Sudeste Asiático, outra região endêmica no mundo 5,32,33,34.

Nesta investigação, o uso de óbitos por dengue como evento sentinela da qualidade da assistência demonstrou que, em se tratando de um desfecho evitável, é possível evidenciarem-se os aspectos problemáticos, seja por insuficiência, inaptidão ou negligência, envolvidos na ocorrência das mortes. Embora alguns casos graves de dengue possam levar ao óbito, mesmo com atenção adequada e oportuna, justificando as taxas esperadas de letalidade até $1 \%$, os altos índices verificados no Brasil não podem ser explicados sem que se leve em consideração a qualidade da assistência prestada ao paciente com a doença.

Como limitações do estudo, pode-se apontar a dificuldade de realização das entrevistas com os profissionais médicos em alguns serviços de saúde. No caso das chefias de setor, dois profissionais estavam ausentes no período de coleta de dados (licença médica e férias); quanto aos profissionais responsáveis pela assistência, foram necessárias várias visitas aos serviços para sua localização, além de três questionários não terem sido devolvidos. Porém, não houve comprometimento da análise do grau de implantação das ações, visto que as respostas dos profissionais foram coincidentes em muitos aspectos e 
iam saturando os itens do questionário quando da análise dos dados. Por fim, não foi possível localizar ou ter acesso a alguns prontuários médicos de unidades básicas de saúde e policlínicas; ademais, em um dos casos, ocorrido no Hospital Municipal, B) (HM, B), o tempo de permanência do paciente no serviço foi de apenas 15 minutos, não permitindo avaliar a qualidade da assistência prestada, com base nos registros do prontuário médico.

\section{Conclusão}

Os resultados do estudo sinalizam que tanto os aspectos da estrutura e processo dos serviços, quanto o acesso dos usuários à rede de saúde, não se constituíram nos principais problemas para a qualidade da assistência aos pacientes nos casos avaliados. Os serviços de saúde avaliados tinham condições de trabalho ao menos parcialmente adequadas para desenvolver as ações e práticas essenciais para o tratamento da dengue. Logo,

\section{Resumo}

Apesar dos meios para assistência adequada aos pacientes de dengue na rede de saúde, os índices de letalidade pela doença mantêm-se altos no Brasil. Visando a identificar implicações para ocorrência do óbito, este estudo avaliou a qualidade da assistência conforme grau de implantação das ações, qualidade técnicocientífica da atenção e acesso aos serviços de saúde, em dois municípios do Nordeste do Brasil. Realizou-se pesquisa avaliativa, do tipo análise de implantação, tendo o óbito por dengue como evento sentinela da qualidade da assistência. Para avaliação do grau de implantação e qualidade da atenção, pontuaram-se os critérios das entrevistas e análise dos prontuários; para o acesso, procedeu-se à análise temática. Quanto à estrutura e ao processo, os serviços de saúde encontram-se parcialmente adequados (70\%). Não foram encontradas barreiras geográficas e econômicas que justificassem a ocorrência dos óbitos. A qualidade técnico-científica não alcançou a adequação nos municípios (46\% e 30\%) e nos serviços avaliados, observando-se insuficiência no manejo clínico da dengue nos serviços de saúde.

Dengue; Avaliação em Saúde; Assistência à Saúde; Vigilância de Evento Sentinela tais aspectos não deveriam ser referidos como justificativa para a ocorrência dos óbitos, mesmo ressalvando-se a necessidade de investimento no componente de apoio laboratorial.

Os achados demonstraram que os óbitos por dengue que compuseram o presente estudo deveram-se, sobretudo, à insuficiência na observação dos protocolos de manejo clínico da doença, principalmente quanto ao estadiamento dos casos e utilização dos exames clínicos, nos serviços de saúde avaliados. O não seguimento dos procedimentos preconizados para os casos graves de dengue foi observado na inadequação do diagnóstico da doença, na identificação tardia dos sinais de agravamento, na condução do tratamento e na referência inadequada dos casos, conforme o registro dos prontuários médicos.

O monitoramento dos casos graves e a investigação sistemática dos óbitos por dengue nos serviços de saúde é recomendável, pois permitirá identificar problemas e atuar prontamente, visando a melhorar a qualidade da assistência aos pacientes e a evitar desfechos indesejáveis.

\section{Colaboradores}

A. C. Figueiró participou de todas as etapas da pesquisa e da elaboração do artigo. Z. M. A. Hartz participou de todas as etapas da elaboração do artigo. C. A. A. Brito participou da pesquisa, da análise e interpretação dos resultados. I. Samico participou da pesquisa, da redação e revisão crítica do artigo. N. T. Siqueira Filha participou da pesquisa, da análise e interpretação dos resultados. G. Cazarin participou da pesquisa, da análise e interpretação dos resultados. C. Braga participou da redação e revisão crítica do artigo. E. A. P. Cesse participou da redação e revisão crítica do artigo.

\section{Agradecimentos}

Os autores agradecem à Secretaria de Vigilância em Saúde do Ministério da Saúde pelo financiamento da pesquisa. 


\section{Referências}

1. World Health Organization. Dengue and dengue hemorrhagic fever. Fact sheet, 117. http://www. who.int/mediacentre/factsheets/fs117/en/index. html (acessado em 14/Dez/2009).

2. Secretaria de Vigilância em Saúde, Ministério da Saúde. Dengue - casos da doença no Brasil; 2010. http://portal.saude.gov.br/portal/saude/profis sional/visualizar_texto.cfm?idtxt=31131 (acessado em 30/Nov/2010).

3. Teixeira MG, Costa MCN, Barreto F, Barreto ML. Dengue: twenty-five years since reemergence in Brazil. Cad Saúde Pública 2009; 25 Suppl 1:S7-18.

4. Câmara FP, Theophilo RLG, Santos GT, Pereira SRFG, Câmara DCP, Matos RRC. Estudo retrospectivo (histórico) da dengue no Brasil: características regionais e dinâmicas. Rev Soc Bras Med Trop 2007; 40:192-6.

5. Ministério da Saúde/Fundação Nacional de Saúde. Programa Nacional de Controle da Dengue (PNCD). Brasília: Fundação Nacional de Saúde; 2002.

6. Torres ME. La prevención de la mortalidad por dengue: un espacio y un reto para la atención primaria de salud. Rev Panam Salud Pública 2006; 20:60-74.

7. Singhi S, Kisson N, Bansal A. Dengue and dengue hemorrhagic fever: management issues in an intensive care unit. J Pediatr 2007; 83 Suppl 2: S22-35.

8. Donabedian A. Evaluating the quality of medical care. Milbank Q 1966; 44:166-203.

9. Donabedian A. The seven pillars of quality. Arch Pathol Lab Med 1990; 114:1115-8.

10. Vuori H. A qualidade da saúde. Divulg Saúde Debate 1991; 3:17-25.

11. Uchimura KY, Bosi MLM. Qualidade e subjetividade na avaliação de programas e serviços de saúde. Cad Saúde Pública 2002; 18:1561-9.

12. Secretaria de Vigilância em Saúde, Ministério da Saúde. Dengue, diagnóstico e manejo clínico. Brasília: Ministério da Saúde; 2007.

13. Frenk J. El concepto y la medición de accesibilidad. Salud Pública Méx 1985; 27:438-53.

14. Champagne F, Brousselle A, Hartz Z, Contandriopoulos AP. L'analyse de l'implantation. In: Brousselle A, Champagne F, Contandriopoulos AP, Hartz Z, editors. L'évaluation: concepts et méthodes. Montréal: Les Presses de l'Université de Montréal; 2009. p. 225-48.

15. Yin RK. Estudo de caso: planejamento e método. 3a Ed. Porto Alegre: Bookman; 2005.

16. The Joint Commission. Sentinel event policy and procedures 2007 Available from: http://www.joint commission.org/NR/rdonlyres/F84F9DC6-A5DA490F-A91F-A9FCE26347C4/0/SE_chapter_july07. pdf (accessado em 11/Nov/2009).

17. Rutstein DD, Berenberg W, Chalmers TC, Child CG, Fishman AP, Perrin EB. Measuring the quality of medical care: a clinical method. N Engl J Med 1976; 294:582-8.
18. Hartz ZMA, Champagne F, Leal MC, Contandriopoulos AP. Mortalidade infantil "evitável" em duas cidades do nordeste do Brasil: indicador de qualidade do sistema local de saúde. Rev Saúde Pública 1996; 30:310-8.

19. Australian Institute of Health and Welfare/Australian Commission on Safety and Quality in Health Care. Sentinel events in Australian public hospitals 2004-05. http://www.aihw.gov.au/publica tion-detail/?id=6442468005 (accessado em 11/ Nov/2009).

20. Dipartimento della Qualitá, Direzione Generale della Programmazione Sanitaria, dei Livelli di Assistenza e dei Principi Etici di Sistema, Ufficio III, Ministero del Lavoro, della Salute e delle Politiche Sociali. Osservatorio Nazionale sugli Eventi Sentinella. Protocollo per il monitoraggio degli eventi sentinella. http://www.salute.gov.it/imgs/C_17_ pagineAree_238_listaFile_itemName_0_file.pdf (acessado em 11/Nov/2009).

21. Lopes RM, Vieira-da-Silva LV, Hartz ZMA. Teste de uma metodologia para avaliar a organização, acesso e qualidade técnica do cuidado na atenção à diarréia na infância. Cad Saúde Pública 2004; 20 Suppl 2:S283-97.

22. Medina MG. O contexto local, a organização da atenção primária e a implementação de redes integradas de atenção à saúde: resultados da avaliação de dois estudos de caso [Tese de Doutorado]. Salvador: Instituto de Saúde Coletiva, Universidade Federal da Bahia; 2006.

23. Bellato R, Araújo LFS, Castro P. O itinerário terapêutico como uma tecnologia avaliativa da integralidade em saúde. In: Pinheiro R, Silva-Junior AG, Mattos RA, organizadores. Atenção básica e integralidade: contribuições para estudos de práticas avaliativas em saúde. Rio de Janeiro: Centro de Estudos e Pesquisa em Saúde Coletiva, Instituto de Medicina Social, Universidade do Estado do Rio de Janeiro/ABRASCO; 2008. p. 167-87.

24. Braun V, Clarke V. Using thematic analysis in psychology. Qualitative Research in Psychology 2006; 3:77-101.

25. Secretaria de Vigilância em Saúde, Ministério da Saúde. Diretrizes nacionais para prevenção e controle de epidemias de dengue. Série A. Normas e manuais técnicos. Brasília: Ministério da Saúde; 2009.

26. Cavalcante MGS, Samico I, Frias PG, Vidal AS. Análise de implantação das áreas estratégicas da atenção básica nas equipes de Saúde da Família em municípios de uma Região Metropolitana do Nordeste Brasileiro. Rev Bras Saúde Matern Infant 2006; 6:437-45. 
27. Gusmão-Filho FAR. Análise de implantação da Política de Qualificação da Atenção à Saúde do Ministério da Saúde - Política Qualisus - em três hospitais do município do Recife [Tese de Doutorado]. Recife: Centro de Pesquisas Aggeu Magalhães, Fundação Oswaldo Cruz; 2008.

28. Samico I, Hartz ZMA, Felisberto E, Carvalho EF Atenção à saúde da criança: uma análise do grau de implantação e da satisfação de profissionais e usuários em dois municípios do estado de Pernambuco, Brasil. Rev Bras Saúde Matern Infant 2005; 5:229-40.

29. Halstead SB, Lum LCS. Assessing the prognosis of dengue-infected patients. F1000 Med Rep 2009; 1:73-6.

30. Hartz ZMA, Contandriopoulos A-P. Integralidade da atenção e integração de serviços de saúde: desafios para avaliar a implantação de um "sistema sem muros". Cad Saúde Pública 2004; 20 Suppl 2:S331-6.
31. Frias PG, Lira PIC, Vidal AS, Vanderlei LC. Vigilância de óbitos infantis como indicador da efetividade do sistema de saúde: estudo em um município do interior do Nordeste brasileiro. J Pediatr (Rio J.) 2002; 78:509-16.

32. Teixeira MG, Costa MCN, Barreto ML, Mota E. Dengue and dengue hemorrhagic fever epidemics in Brazil: what research is needed based on trends, surveillance, and control experiences? Cad Saúde Pública 2005; 21:1307-15.

33. Kalayanarooj S, Rimal HS, Andjaparidze A, Vatcharasaevee V, Nisalak A, Jarman RG, et al. Clinical intervention and molecular characteristics of a dengue hemorrhagic fever outbreak in Timor Leste, 2005. Am J Trop Med Hyg 2007; 77:534-7.

34. Khun S, Manderson L. Health seeking and access to care for children with suspected dengue in Cambodia: an ethnographic study. BMC Public Health 2007; 7:262-1.

Recebido em 14/Dez/2010

Versão final reapresentada em 20/Ago/2011

Aprovado em 15/Set/2011 